\title{
Severe Hepaticojejunostomy Dehiscence Treatment using a Shape- Modifying Covered Metal Stent with Single-Balloon Enteroscope
}

\author{
Tadahisa Inoue, Rena Kitano, Kiyoaki Ito, Masashi Yoneda \\ Department of Gastroenterology, Aichi Medical University, Aichi, Japan
}
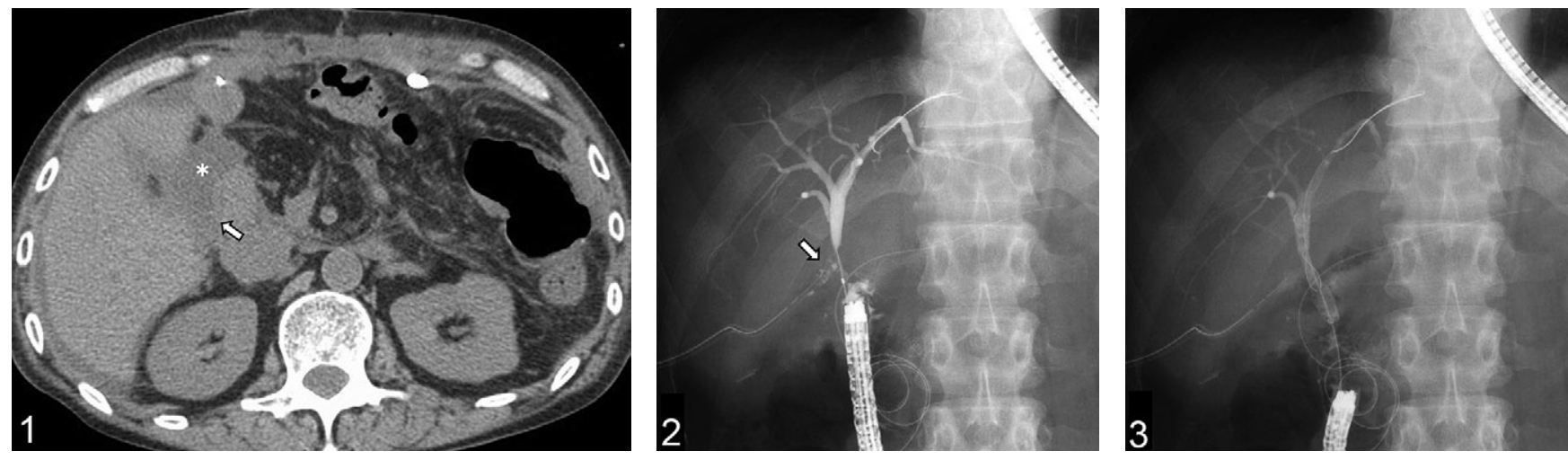

A 54-year-old man, who underwent pancreaticoduodenectomy, had persistent postoperative high fever and abdominal pain. Nine days postoperatively, computed tomography revealed a fluid collection (Fig 1, asterisks) near the hepaticojejunostomy (Fig. 1, arrow). Consequently, percutaneous drainage was performed and bile was observed passing through the drainage tube. After drainage, his fever and laboratory data improved. However, bile discharge through the tube gradually increased. Endoscopic retrograde cholangiography, using a short-type single-balloon enteroscope (SBE) (SIF-H290S; Olympus Medical Systems, Tokyo, Japan), performed 27 days postoperatively revealed severe dehiscence of the anastomosis; an intraperitoneal cavity was seen beyond the jejunal portion of the anastomosis. A 0.025-inch guidewire was inserted into the anastomosis site to reach the bile duct. Cholangiography revealed bile leakage between the bile duct and the jejunum (Fig. 2). Therefore, a shape-modifying fully covered metal stent (MS) (BONASTENT M-Intraductal; Standard SciTech Inc., Seoul, Korea), having a unique structure that prevents tissue hyperplasia and early migration [1], was placed across the leakage site, and from the hepatic duct to the jejunum (Fig. 3). Discharge into the percutaneous drainage tube rapidly ceased after endoscopic intervention, without any adverse event. It was removed one month after the procedure, and no recurrence was observed during the follow-up period.

Our procedure may offer a less invasive, but effective treatment option for severe hepaticojejunostomy dehiscence. To our knowledge, only plastic stent placement has been reported as endoscopic treatment of postoperative bile leakage after hepaticojejunostomy [2,3]. However, plastic stents might be insufficient for covering the leakage site in severe dehiscence cases. Covered MS can be used with short-type SBEs, which have a working length of $1520 \mathrm{~mm}$ and a working channel of $3.2 \mathrm{~mm}$ [4]. Additionally, a shape-modifying, fully covered MS may be more suitable for this type of treatment, as it minimizes stent-induced injury and early migration.

Corresponding author: Tadahisa Inoue, tinoue-tag@umin.ac.jp

Conflicts of interest: None to declare.

\section{REFERENCES}

1. Moon JH, Choi HJ, Koo HC, et al. Feasibility of placing a modified fully covered self-expandable metal stent above the papilla to minimize stent-induced bile duct injury in patients with refractory benign biliary strictures (with videos). Gastrointest Endosc 2012;75:1050-1055. doi:10.1016/j.gie.2012.01.016

2. Matsumoto K, Tsutsumi K, Kato H, et al. Efficacy of endoscopic treatment using double-balloon enteroscopy for postoperative bile leakage in patients with hepaticojejunoscopy. Endosc Int Open 2018;6:E211-E216. doi:10.1055/s-0043-125143

3. Nagai K, Yane K, Katanuma A, et al. Successful less-invasive endoscopic treatment for bile leakage from choledochojejunostomy site using short-type single-balloon enteroscopy. Endoscopy 2016;48:E140-E141. doi:10.1055/s-0042-105208

4. Moreels TG. Update in enteroscopy: New devices and new indications. Dig Endosc 2018;30:174-181. doi:10.1111/den.12920 